\title{
Multiobjective Optimization, Scalarization, and Maximal Elements of Preorders
}

\author{
Paolo Bevilacqua, ${ }^{1}$ Gianni Bosi $\mathbb{D}{ }^{2}{ }^{2}$ and Magalì Zuanon ${ }^{3}$ \\ ${ }^{1}$ DIA, Università di Trieste, 34127 Trieste, Italy \\ ${ }^{2}$ DEAMS, Università di Trieste, 34127 Trieste, Italy \\ ${ }^{3}$ DEM, Università di Brescia, 25122 Brescia, Italy \\ Correspondence should be addressed to Gianni Bosi; gianni.bosi@deams.units.it
}

Received 31 July 2017; Accepted 17 December 2017; Published 28 January 2018

Academic Editor: Simeon Reich

Copyright (C) 2018 Paolo Bevilacqua et al. This is an open access article distributed under the Creative Commons Attribution License, which permits unrestricted use, distribution, and reproduction in any medium, provided the original work is properly cited.

\begin{abstract}
We characterize the existence of (weak) Pareto optimal solutions to the classical multiobjective optimization problem by referring to the naturally associated preorders and their finite (Richter-Peleg) multiutility representation. The case of a compact design space is appropriately considered by using results concerning the existence of maximal elements of preorders. The possibility of reformulating the multiobjective optimization problem for determining the weak Pareto optimal solutions by means of a scalarization procedure is finally characterized.
\end{abstract}

\section{Introduction}

It is very well known that multiobjective optimization (see, e.g., Miettinen [1] and Ehrgott [2]) allows choosing among various available options in the presence of more than one agent (or criterion), and therefore it represents a popular and important tool which appears in many different disciplines. This is the case, for example, of design engineering (see, e.g., Das [3] and Pietrzak [4]), portfolio selection (see, e.g., Xidonas et al. [5]), economics and risk-sharing (see, e.g., Chateauneuf et al. [6] and Barrieu an Scandolo [7]), and insurance theory (see, e.g., Asimit et al. [8]).

The multiobjective optimization problem (MOP) is usually formulated by means of the standard notation (needless to say, this formulation of the multiobjective optimization problem is equivalent, "mutatis mutandis," to $\min _{x \in X}\left[f_{1}(x), \ldots, f_{m}(x)\right]=\min _{x \in X} \mathbf{f}(x), m \geq 2$; we use the approach with the maximum for the sake of convenience):

$$
\max _{x \in X}\left[u_{1}(x), \ldots, u_{m}(x)\right]=\max _{x \in X} \mathbf{u}(x), \quad m \geq 2,
$$

where $X$ is the choice set (or the design space), $u_{i}$ is the decision function (in this case a utility function) associated with the $i$ th individual (or criterion), and $\mathbf{u}: X \mapsto \mathbb{R}^{m}$ is the vector-valued function defined by $\mathbf{u}(x)=\left(u_{1}(x), \ldots, u_{m}(x)\right)$ for all $x \in X$.

An element $x_{0} \in X$ is a (weak) Pareto optimal solution to problem (1), for every $x \in X$, if $u_{i}\left(x_{0}\right) \leq u_{i}(x)$ for every $i \in\{1, \ldots, m\}$; then $u_{i}\left(x_{0}\right)=u_{i}(x)$ for every index $i$ (respectively, for every $x \in X$, if $u_{i}\left(x_{0}\right) \leq u_{i}(x)$ for every $i \in\{1, \ldots, m\}$; then $u_{i}\left(x_{0}\right)=u_{i}(x)$ for at least one index $\left.i\right)$. In this case, the point $x_{0} \in X$ is said to be (weakly) Pareto optimal or a (weakly) efficient point for (MOP). Usually, $X$ is a subset of $\mathbb{R}^{n}$ and concavity restrictions are posed on the functions $u_{i}$ (see, e.g., Ehrgott and Nickel [9]). In this case, an appropriate scalarized problem can be considered to determine Pareto optimal solutions (see Miettinen [1, Theorems 3.4.5 and 3.5.4]). Further, robust multiobjective optimization has been also considered in the literature (see, e.g., Bokrantz and Fredriksson [10]).

It should be noted that Pareto optimality can be also considered by starting from a family $\left\{\Im_{i}\right\}_{i \in\{1, \ldots, m\}}$ of not necessarily total preorders on a set $X$ (see, e.g., d'Aspremont and Gevers [11]).

In this paper we approach the multiobjective optimization problem (1) by referring to the preorders which are naturally associated with this problem. This means that, for 
determining the Pareto optimal solutions, we introduce the preorder $\preccurlyeq_{\mathbf{u}}$ on $X$ defined, for all $x, y \in X$, by

$$
\begin{gathered}
x \preccurlyeq_{\mathbf{u}} y \Longleftrightarrow \\
{\left[u_{i}(x) \leq u_{i}(y) \quad \forall i \in\{1, \ldots, m\}\right],}
\end{gathered}
$$

and, for determining the weak Pareto optimal solutions, we refer to the preorder $\Im_{\mathbf{u}}^{w}$ on $X$ defined, for all $x, y \in X$, by

$$
\begin{aligned}
& \quad x \precsim_{\mathbf{u}}^{w} y \Longleftrightarrow \\
& {\left[\left(u_{i}(x)=u_{i}(y) \forall i \in\{1, \ldots, m\}\right)\right.} \\
& \left.\quad \text { or }\left(u_{i}(x)<u_{i}(y) \forall i \in\{1, \ldots, m\}\right)\right] .
\end{aligned}
$$

The consideration that an element $x_{0} \in X$ is a (weak) Pareto optimal solution to problem (1) if and only if $x_{0} \in X$ is a maximal element for the preorder $\precsim_{\mathbf{u}}\left(\varsigma_{\mathbf{u}}^{w}\right.$, respectively $)$ and the observation that the function $\mathbf{u}=\left(u_{1}, \ldots, u_{m}\right): X \mapsto \mathbb{R}^{m}$ is a (Richter-Peleg) multiutility representation of the preorder $\Im_{\mathbf{u}}\left(\Im_{\mathbf{u}}^{w}\right.$, respectively) allow us to present various results concerning the existence of solutions to the multiobjective optimization problem, also in the classical case when the design space is a compact topological space. We recall that the concept of a (finite) multiutility representation of a preorder was introduced and studied by Ok [12] and Evren and Ok [13], while Richter-Peleg multiutility representations were introduced by Minguzzi [14] and then studied by Alcantud et al. [15].

The consideration of a compact design space allows us to use classical results concerning the existence of maximal elements for preorders on compact spaces (see RodríguezPalmero and García-Lapresta [16] and Bosi and Zuanon [17]). We also address the scalarization problem by using classical results in Decision Theory related to potential optimality of maximal elements (see Podinovski $[18,19]$ ). In particular, we refer to a classical theorem of White [20], according to which every maximal element for a preorder is determined by maximizing an order-preserving function (provided that an order-preserving function exists). In particular, we show that when considering the multiobjective optimization problem (1) in order to determine the weak Pareto optimal solution, this problem can be reformulated as an equivalent one in a such a way that every weak Pareto optimal solution is determined by maximizing an objective function.

It should be noted that the results presented are fairly general, and we do not impose any restrictions neither to the choice set $X$, which usually is assumed to coincide with $\mathbb{R}^{n}$, nor to the real-valued functions $u_{i}$ that are usually assumed to be concave in the literature.

\section{Notation and Preliminaries}

Let $X$ be a nonempty set (decision space) and denote by $\lesssim$ a preorder (i.e., a reflexive and transitive binary relation) on $X$. If in addition $\lesssim$ is antisymmetric, then it is said to be an order. As usual, $\prec$ denotes the strict part of $\precsim$ (i.e., for all $x, y \in X$, $x \prec y$ if and only if $(x \preceq y)$ and not $(y \preceq x))$. Furthermore, $\sim$ stands for the indifference relation (i.e., for all $x, y \in X$, $x \sim y$ if and only if $(x \preceq y)$ and $(y \preceq x))$. We have that $\sim$ is an equivalence relation on $X$. We denote by $\Im_{\sim}$ the quotient order on the quotient set $X_{\mid \sim}$ (i.e., for all $x, y \in X,[x] \nwarrow_{\mid \sim}[y]$ if and only if $x \preceq y$, where $[x]=\{z \in X: z \sim x\}$ is the indifference class associated with $x \in X$ ).

For every $x \in X$, we set

$$
\begin{aligned}
& l(x)=\{z \in X \mid z \prec x\}, \\
& i(x)=\{z \in X \mid x \precsim z\} .
\end{aligned}
$$

Given a preordered set $(X, \precsim)$, a point $x_{0} \in X$ is said to be a maximal element of $X$ if for no $z \in X$ it occurs that $x_{0}<z$. In the sequel we shall denote by $X_{M}^{s}$ the set of all the maximal elements of a preordered set $(X, \precsim)$. Please observe that $X_{M}$ can be empty.

Denote by $\bowtie$ the incomparability relation associated with a preorder $\preccurlyeq$ on a set $X$ (i.e., for all $x, y \in X, x \bowtie y$ if and only if not $(x \preceq y)$ and not $(y \lesssim x))$. be

We recall that a function $u:(X, \preceq) \mapsto(\mathbb{R}, \leq)$ is said to

(1) isotonic or increasing if $x \lessgtr y \Rightarrow u(x) \leq u(y)$ for all $x, y \in X$

(2) strictly isotonic or order-preserving if it is isotonic and, in addition, $x<y \Rightarrow u(x)<u(y)$ for all $x, y \in X$.

Strictly isotonic functions on $(X, \precsim)$ are also called RichterPeleg representations of $\lesssim$ in the economic literature (see, e.g., Richter [21] and Peleg [22]).

Definition 1. A family $\mathbf{U}=\left\{u_{1}, \ldots, u_{m}\right\}$ of (necessarily isotonic) functions $u_{i}:(X, \precsim) \mapsto(\mathbb{R}, \leq)$ is said to be

(1) a finite multiutility representation of the preorder $\lesssim$ on $X$ if, for all $x, y \in X$,

$$
\begin{gathered}
x \precsim y \Longleftrightarrow \\
{\left[u_{i}(x) \leq u_{i}(y), \quad \forall i \in\{1, \ldots, m\}\right] ;}
\end{gathered}
$$

(2) a finite Richter-Peleg multiutility representation of the preorder $\preccurlyeq$ on $X$ if $\mathbf{U}$ is a finite multiutility representation and in addition every function $u_{i} \in \mathrm{U}$ is a Richter-Peleg representation of $\precsim$.

Alcantud et al. [15, Remark 2.3] noticed that a (finite) Richter-Peleg multiutility representation $\mathbf{U}$ of a preorder $\lesssim$ on a set $X$ also characterizes the strict part $\prec$ of $\precsim$, in the sense that, for each $x, y \in X$,

$$
\begin{gathered}
x<y \Longleftrightarrow \\
{[u(x)<u(y), \forall u \in \mathbf{U}] .}
\end{gathered}
$$

Definition 2. Consider the multiobjective optimization problem (1). Then a point $x_{0} \in X$ is said to be

(1) Pareto optimal with respect to the function $\mathbf{u}=\left(u_{1}\right.$, $\left.\ldots, u_{m}\right): X \mapsto \mathbb{R}^{m}$ if for no $x \in X$ it occurs that $u_{i}\left(x_{0}\right) \leq u_{i}(x)$ for all $i \in\{1, \ldots, m\}$ and at the same time $u_{i}\left(x_{0}\right)<u_{i}(x)$ for at least one index $i$; 
(2) weakly Pareto optimal with respect to the function $\mathbf{u}=$ $\left(u_{1}, \ldots, u_{m}\right): X \mapsto \mathbb{R}^{m}$ if for no $x \in X$ it occurs that $u_{i}\left(x_{0}\right)<u_{i}(x)$ for all $i \in\{1, \ldots, m\}$.

Definition 3. The set of all (weakly) Pareto optimal elements with respect to the function $\mathbf{u}=\left(u_{1}, \ldots, u_{m}\right): X \mapsto \mathbb{R}^{m}$ will be denoted by $X_{\mathbf{u}}^{\mathrm{Par}}\left(X_{\mathbf{u}}^{w \mathrm{Par}}\right.$, respectively).

It is clear that $X_{\mathbf{u}}^{\mathrm{Par}} \subset X_{\mathbf{u}}^{w \text { Par }}$ for every positive integer $m$, every nonempty set $X$, and every function $\mathbf{u}=\left(u_{1}, \ldots, u_{m}\right)$ : $X \mapsto \mathbb{R}^{m}$.

Definition 4. Consider the multiobjective optimization problem (1). Then we introduce the preorders $\precsim_{\mathbf{u}}$ and $\precsim_{\mathbf{u}}^{w}$ on $X$ defined as follows for all $x, y \in X$ :

(1) $x \lesssim_{\mathbf{u}} y \Leftrightarrow\left[u_{i}(x) \leq u_{i}(y)\right.$ for all $\left.i \in\{1, \ldots, m\}\right]$.

(2) $x \nwarrow_{\mathbf{u}}^{w} y \Leftrightarrow\left[\left(u_{i}(x)=u_{i}(y)\right.\right.$ for all $\left.i \in\{1, \ldots, m\}\right)$ or $\left(u_{i}(x)<u_{i}(y)\right.$ for all $\left.\left.i \in\{1, \ldots, m\}\right)\right]$.

Remark 5. Notice that the indifference relation $\sim_{\mathbf{u}}$ and the strict part $\prec_{\mathbf{u}}$ of the preorder $\precsim_{\mathbf{u}}$, as well as the indifference relation $\sim_{\mathbf{u}}^{w}$ and the strict part $\prec_{\mathbf{u}}^{w}$ of the preorder $\precsim_{\mathbf{u}}^{w}$, are defined as follows, for all $x, y \in X$ :

$$
\begin{aligned}
& x \sim{ }_{\mathbf{u}} y \Longleftrightarrow \\
& x \sim \sim_{\mathbf{u}}^{w} y \Longleftrightarrow \\
& {\left[u_{i}(x)=u_{i}(y) \forall i \in\{1, \ldots, m\}\right] \text {, }} \\
& x \prec_{\mathbf{u}} x \Longleftrightarrow \\
& {\left[u_{i}(x) \leq u_{i}(y) \forall i \in\{1, \ldots, m\}\right]} \\
& \text { and there exists } i \in\{1, \ldots, m\} \\
& \text { such that } u_{i}(x)<u_{i}(y) \text {, } \\
& x \prec_{\mathbf{u}}^{w} y \Longleftrightarrow \\
& {\left[u_{i}(x)<u_{i}(y) \forall i \in\{1, \ldots, m\}\right] .}
\end{aligned}
$$

Definition 6. A preorder $\lesssim$ on a topological space $(X, \tau)$ is said to be

(1) upper semiclosed if $i(x)=\{z \in X \mid x \precsim z\}$ is a closed subset of $X$ for every $x \in X$;

(2) upper semicontinuous if $l(x)=\{z \in X \mid z \prec x\}$ is an open subset of $X$ for every $x \in X$.

While it is guaranteed that a preorder $\lesssim$ on a compact topological space $(X, \tau)$ has a maximal element provided that $\precsim$ is either upper semiclosed (see Ward Jr. [23, Theorem 1]) or upper semicontinuous (see the theorem in Bergstrom [24]), a characterization of the existence of a maximal element for a preorder on a compact topological space $(X, \tau)$ was presented by Rodríguez-Palmero and García-Lapresta [16].

Definition 7 (see Rodríguez-Palmero and García-Lapresta [16, Definition 4]). A preorder $\lesssim$ on a topological space $(X, \tau)$ is said to be transfer transitive lower continuous if for every element $x \in X$ which is not a maximal element of $\precsim$ there exist an element $y \in X$ and a neighbourhood $\mathcal{N}(x)$ of $x$ such that $y \prec z$ implies that $\mathcal{N}(x) \prec z$ for all $z \in X$.

Theorem 8 (see Rodríguez-Palmero and García-Lapresta [16, Theorem 3]). A preorder $\lesssim$ on a compact topological space $(X, \tau)$ has a maximal element if and only if it is transfer transitive lower continuous.

We recall that a real-valued function $u$ on a topological space $(X, \tau)$ is said to be upper semicontinuous if $u^{-1}(]-$ $\infty, \alpha[)=\{x \in X: u(x)<\alpha\}$ is an open set for all $\alpha \in \mathbb{R}$. A popular theorem guarantees that an upper semicontinuous real-valued function attains its maximum on a compact topological space.

As usual, for a real-valued function $u$ on a nonempty set $X$, we denote by $\arg \max u$ the set of all the points $x \in X$ such that $u$ attains its maximum at $x$ (i.e., $\arg \max u=\{x \in$ $X: u(z) \leq u(x)$ for all $z \in X\})$.

\section{Existence of Maximal Elements and Pareto Optimality}

A finite family $\mathbf{U}=\left\{u_{1}, \ldots, u_{m}\right\}$ of real-valued functions on a nonempty set $X$ gives rise to a preorder $\lesssim$ on $X$ which admits precisely the (Richter-Peleg) multiutility representation U. It is easy to relate the maximal elements of such a preorder $\lesssim$ to the solutions of the associated multiobjective optimization problem (1).

Theorem 9. Let $\lesssim$ be a preorder on a set X. Then the following statements hold:

(1) If admits a finite multiutility representation $\mathbf{U}=\left\{u_{1}\right.$, $\left.\ldots, u_{m}\right\}$ then $X_{\mathbf{u}}^{\text {Par }}=X_{M}^{\lessgtr}$.

(2) If $\lesssim$ admits a finite Richter-Peleg multiutility representation $\mathbf{U}=\left\{u_{1}, \ldots, u_{m}\right\}$ then $X_{\mathbf{u}}^{w \text { Par }}=X_{M}^{\precsim}$.

Proof. Assume that the preorder $\lesssim$ on $X$ admits a finite (Richter-Peleg) multiutility representation $\mathbf{U}=\left\{u_{1}, \ldots, u_{m}\right\}$. In order to show that $X_{\mathbf{u}}^{\mathrm{Par}} \subset X_{M}^{\lessgtr}\left(X_{\mathbf{u}}^{w \text { Par }} \subset X_{M}^{\Im}\right)$, consider, by contraposition, an element $x_{0} \notin X_{M}^{\Im}$. Then there exists an element $x \in X$ such that $x_{0} \prec x$, or equivalently $u_{i}\left(x_{0}\right) \leq$ $u_{i}(x)$ for all $i \in\{1, \ldots, m\}$ with an index $i \in\{1, \ldots, m\}$ such that $u_{i}\left(x_{0}\right)<u_{i}(x)$ (respectively, $u_{i}\left(x_{0}\right)<u_{i}(x)$ for all $i \in\{1, \ldots, m\})$. Then we have that $x_{0}$ is not (weakly) Pareto optimal. In a perfectly analogous way it can be shown that $X_{M}^{\lessgtr} \subset X_{\mathbf{u}}^{\mathrm{Par}}\left(X_{M}^{\lessgtr} \subset X_{\mathbf{u}}^{w P \mathrm{ar}}\right)$. Hence, the proof is complete.

The following proposition is an immediate consequence of Definition 4.

Proposition 10. Consider the multiobjective optimization problem (1). Then $\mathbf{U}=\left\{u_{1}, \ldots, u_{m}\right\}$ is a finite (Richter-Peleg) multiutility representation of the preorder $\lesssim_{\mathbf{u}}\left(\S_{\mathbf{u}}^{w}\right.$, respectively).

From Theorem 9 and Proposition 10, we immediately arrive at the following proposition. 
Proposition 11. Consider the multiobjective optimization problem (1). The following conditions are equivalent on a point $x_{0} \in X$ :

(i) $x_{0}$ is (weakly) Pareto optimal with respect to the function $\mathbf{u}=\left(u_{1}, \ldots, u_{m}\right)$.

(ii) $x_{0}$ is maximal with respect to the preorder $\precsim_{\mathbf{u}}\left(\Im_{\mathbf{u}}^{w}\right)$ on $X$.

\section{Multiobjective Optimization on Compact Spaces}

The following theorem provides a characterization of the existence of Pareto optimal solutions to the multiobjective optimization problem (1) in terms of compactness of the choice set and appropriate semicontinuity conditions of the strict parts of the naturally associated preorders.

Theorem 12. Consider the multiobjective optimization problem (1). The following conditions are equivalent:

(i) $X_{\mathbf{u}}^{\text {Par }}\left(X_{\mathbf{u}}^{\text {war }}\right)$ is nonempty.

(ii) There exists a compact topology $\tau$ on $X$ and an upper semiclosed preorder $\lesssim$ on $X$ such that $\prec_{\mathbf{u}} \subset \prec\left(\prec_{\mathbf{u}}^{w} \subset \prec\right)$.

(iii) There exists a compact topology $\tau$ on $X$ such that $\lesssim_{\mathbf{u}}\left(\S_{\mathbf{u}}^{w}\right)$ is upper semicontinuous.

Proof. (i) $\Rightarrow$ (ii). Since $X_{\mathbf{u}}^{\text {Par }}\left(X_{\mathbf{u}}^{w \text { Par }}\right)$ is nonempty, we have that $X_{M}^{\varsigma_{\mathrm{u}}}\left(X_{M}^{\varsigma_{\mathrm{u}}^{w}}\right)$ is nonempty by Proposition 11. Therefore, condition (ii) is verified by Bosi and Zuanon [17, Corollary 3.2 , (i) $\Rightarrow$ (ii)].

(ii) $\Rightarrow$ (i). Since $\lesssim$ is an upper semiclosed preorder on compact topological space $(X, \tau)$, from Ward Jr. [23, Theorem 1]. Therefore, also $\lessgtr_{\mathbf{u}}\left(\lesssim_{\mathbf{u}}^{w}\right)$ has a maximal element due to the fact that $\prec_{\mathbf{u}} c \prec\left(\prec_{\mathbf{u}}^{w} \subset \prec\right)$.

(i) $\Leftrightarrow$ (iii). See Alcantud [25, Theorem $4,(a) \Leftrightarrow(b)]$.

Hence, the proof is complete.

Corollary 13. Consider the multiobjective optimization problem (1) where $X$ is endowed with a compact topology $\tau$. Then $X_{\mathbf{u}}^{\text {Par }}$ is nonempty provided that there exist a positive integer $n$ and a function $\mathbf{u}^{\prime}=\left(u_{1}^{\prime}, \ldots, u_{n}^{\prime}\right): X \mapsto \mathbb{R}^{n}$ with all the real-valued functions $u_{i}^{\prime}(i \in\{1, \ldots, n\})$ upper semicontinuous, such that the following condition is verified:

(i) For all $x, y \in X, x \prec_{\mathbf{u}} y$ implies that $u_{i}(x) \leq u_{i}(y)$ for all $i \in\{1, \ldots, n\}$ and there exists $i \in\{1, \ldots, n\}$ such that $u_{i}(x)<u_{i}(y)$.

Proof. By Theorem 9 and Proposition 11, we have that $X_{\mathbf{u}}^{\mathrm{Par}}=X_{M}^{\Im_{\mathbf{u}}}$ is nonempty provided that there exists an upper semiclosed preorder $\lesssim$ on $(X, \tau)$ such that $\prec_{\mathbf{u}} \subset \prec$. Let $\mathbf{u}^{\prime}=$ $\left(u_{1}^{\prime}, \ldots, u_{n}^{\prime}\right): X \mapsto \mathbb{R}^{n}$ be a function with the indicated properties. Define a preorder $\lesssim$ on $X$ by

$$
\begin{gathered}
x \preceq y \Longleftrightarrow \\
{\left[u_{i}^{\prime}(x) \leq u_{i}^{\prime}(y) \forall i \in\{1, \ldots, n\}\right]} \\
\quad(x, y \in X) .
\end{gathered}
$$

The preorder $\lesssim$ is upper semiclosed on $(X, \tau)$ since $u_{i}^{\prime}$ is upper semicontinuous for all $i \in\{1, \ldots, n\}$ and $\mathbf{U}=\left\{u_{1}, \ldots, u_{n}\right\}$ is a (finite) multiutility representation of $\precsim$. Condition (i) precisely means that $\prec_{\mathbf{u}} \subset \prec$. Hence, Theorem 12 , (ii) $\Rightarrow$ (i), applies, and the corollary is proved.

Corollary 14 (see Ehrgott [2, Theorem 2.19]). Consider the multiobjective optimization problem (1) where $X$ is endowed with a compact topology $\tau$ and the real-valued functions $u_{i}(i \in\{1, \ldots, m\})$ are all upper semicontinuous. Then $X_{\mathbf{u}}^{\text {Par }}$ is nonempty.

Proof. This is a particular case of the above Corollary 13, when $m=n$ and $\mathbf{u}^{\prime}=\mathbf{u}$.

As an application of Theorem 8, let us finally present a characterization of the existence of Pareto optimal solution to the multiobjective optimization problem (1) on a compact space. In case that $\lesssim$ is a preorder on a set $X, x$ is an element of $X$, and $A$ is a subset of $X$, the scripture " $A \lesssim x$ " (" $A \prec x$ ") stands for " $z \lesssim x$ for all $z \in A$ " (respectively " $z \prec x$ for all $\left.z \in A^{\prime \prime}\right)$.

Theorem 15. Consider the multiobjective optimization problem (1) where $X$ is endowed with a compact topology $\tau$. Then $X_{\mathbf{u}}^{\text {Par }}$ is nonempty if and only if for every element $x \in X$ which is not Pareto optimal there exist an element $y \in X$ and $a$ neighbourhood $\mathcal{N}(x)$ of $x$ such that, for all $z \in X$, if $y \prec_{\mathbf{u}} z$, then $y^{\prime} \prec_{\mathbf{u}} z$ for all $y^{\prime} \in \mathcal{N}(x)$.

\section{Scalarization and the Representation of All Pareto Optimal Elements}

In this paragraph we address the scalarization of the multiobjective optimization problem under fairly general conditions.

The following theorem was proved by White [20]. Given any maximal element $x_{0}$ relative to a preorder $\lesssim$ on a set $X$, it guarantees the existence of some order-preserving function $u$ attaining its maximum at $x_{0}$.

Theorem 16 (see White [20, Theorem 1]). Let $(X, \precsim)$ be a preordered set and assume that there exists an order-preserving function $u^{\prime}:(X, \precsim) \mapsto(\mathbb{R}, \leq)$. If $X_{M}^{\lessgtr}$ is nonempty, then for every $x_{0} \in X_{M}^{\lessgtr}$ there exists a bounded order-preserving function $u:(X, \precsim) \mapsto(\mathbb{R}, \leq)$ such that $\arg \max u=\left[x_{0}\right]=$ $\left\{z \in X: z \sim x_{0}\right\}$.

The following corollary is an easy consequence of Theorem 16. 
Corollary 17. Consider the multiobjective optimization problem (1). The following conditions are equivalent on a point $x_{0} \in X$ :

(i) $x_{0} \in X_{\mathbf{u}}^{\text {Par }}\left(x_{0} \in X_{\mathbf{u}}^{w \text { Par }}\right)$.

(ii) There exists a bounded real-valued function $u_{x_{0}}$ on $X$ which is order-preserving for the preorder $\precsim_{\mathbf{u}}\left(\S_{\mathbf{u}}^{w}\right)$ on $X$ such that $\arg \max u_{x_{0}}(x)=\left[x_{0}\right]_{\sim_{\mathbf{u}}}\left(\arg \max u_{x_{0}}(x)=\right.$ $\left.\left[x_{0}\right]_{\sim}^{w}\right)$.

Proof. Without loss of generality, we can assume that the functions $u_{i}$ appearing in the multiobjective optimization problem (1) are all bounded. Since $\mathbf{U}=\left\{u_{1}, \ldots, u_{m}\right\}$ is a finite (Richter-Peleg) multiutility representation of the preorder $\precsim_{\mathbf{u}}$ $\left(\Im_{\mathbf{u}}^{w}\right.$, respectively) by Proposition 10 , it is easily seen that the function $u^{*}:=\sum_{h=1}^{m} u_{h}$ is order-preserving for the preorder $\precsim_{\mathbf{u}}\left(\precsim_{\mathbf{u}}^{w}\right.$, respectively). Then we are ready for applying Theorem 16.

The simple proof of the following lemma is left to the reader.

Lemma 18. For any two functions $\mathbf{u}=\left(u_{1}, \ldots, u_{m}\right): X \mapsto$ $\mathbb{R}^{m}$ and $\mathbf{u}^{\prime}=\left(u_{1}^{\prime}, \ldots, u_{m}^{\prime}\right): X \mapsto \mathbb{R}^{m}$, if $\precsim_{\mathbf{u}}=\lesssim_{\mathbf{u}^{\prime}}\left(\precsim_{\mathbf{u}}^{w}=\precsim_{\mathbf{u}^{\prime}}^{w}\right)$, then $X_{\mathbf{u}}^{\text {Par }}=X_{\mathbf{u}^{\prime}}^{\text {Par }}\left(X_{w P a r}^{\mathbf{u}}=X_{w P a r}^{\mathbf{u}^{\prime}}\right)$.

As usual, if $A$ is any nonempty subset of $X$, we denote by $|A|$ the cardinality of $A$.

Theorem 19. Consider the multiobjective optimization problem (1). Then the following conditions are equivalent:

(i) There exist a positive integer $p \leq m$ and a function $\mathbf{u}^{\prime}=\left(u_{1}^{\prime}, \ldots, u_{p}^{\prime}, u_{p+1}^{\prime}, \ldots, u_{m}^{\prime}\right): X \mapsto \mathbb{R}^{m}$ satisfying the following conditions:

(a) $X_{\mathbf{u}}^{w \text { Par }}=X_{\mathbf{u}^{\prime}}^{w \text { Par }}=\bigcup_{i=1}^{p} \arg \max u_{i}^{\prime}$;

(b) $\arg \max u_{i}^{\prime} \cap \arg \max u_{j}^{\prime}=\emptyset$ for all $i \neq j(i, j \in$ $\{1, \ldots, p\})$

(ii) $p=\left|X_{M \mid \sim \sim}^{\Im_{\mathbf{u}}^{w}}\right| \leq m$.

Proof. The implication "(i) $\Rightarrow$ (ii)" is clear. Let us show that also the implication "(ii) $\Rightarrow$ (i)" holds true. Let $p=\left|X_{M \mid \sim \sim}^{乏_{u}^{w}}\right| \leq$ $m$ and $X_{M \mid \sim \sim}^{\Im_{u}^{w}}=\left\{\left[x_{h}\right]\right\}_{h \in\{1, \ldots, p\}}$. Following the proof of White $[20$, Theorem 1], we can define, for every $h \in\{1, \ldots, p\}$ and $x \in X$,

$$
u_{h}^{\prime}(x)= \begin{cases}u_{h}(x) & \text { if } \operatorname{not}\left(x \sim_{\mathbf{u}}^{w} x_{h}\right), \\ \sup u_{h}(x)+\delta_{h} & \text { if } x \sim_{\mathbf{u}}^{w} x_{h},\end{cases}
$$

where $\delta_{1}, \ldots, \delta_{p}$ are positive real numbers. Further, define $u_{k}^{\prime}=u_{k}$ for $k=p+1, \ldots, m$. In this way, the $p$ real-valued functions $u_{1}^{\prime}, \ldots, u_{p}^{\prime}$ are all order-preserving for $\precsim_{\mathbf{u}}^{w}$ such that $\arg \max u_{h}^{\prime}=\left[x_{h}\right]$ for $h=1, \ldots, p$.

It is clear that $\arg \max u_{i}^{\prime} \cap \arg \max u_{j}^{\prime}=\emptyset$ for all $i \neq j(i, j \in\{1, \ldots, p\})$ and that $X_{\mathbf{u}}^{w \operatorname{Par}}=\bigcup_{i=1}^{p} \arg \max u_{i}^{\prime}$.
It remains to show that $X_{\mathbf{u}}^{w \text { Par }}=X_{\mathbf{u}^{\prime}}^{w \text { Par }}$. To this aim, by Lemma 18 it suffices to show that $\precsim_{\mathbf{u}}^{w}=\precsim_{\mathbf{u}^{\prime}}^{w}$ or equivalently that the following property holds for all elements $x, y \in X$ :

(*) $\left[u_{h}(x) \leq u_{h}(y)\right.$ for all $\left.h \in\{1, \ldots, m\}\right] \Leftrightarrow\left[u_{h}^{\prime}(x) \leq\right.$ $u_{h}^{\prime}(y)$ for all $\left.h \in\{1, \ldots, m\}\right]$.

Three cases have to be considered.

(1) $x \in X \backslash X_{M}^{乏_{\mathrm{u}}^{w}}$ and $y \in X \backslash X_{M}^{乏_{\mathrm{u}}^{w}}$. We have that, for every $h \in\{1, \ldots, m\}, u_{h}^{\prime}(x)=u_{h}(x)$ and $u_{h}^{\prime}(y)=u_{h}(y)$. Hence, the above property $(*)$ is obviously verified.

(2) $x \in X_{M}^{\Im_{\mathrm{u}}^{w}}$ and $y \in X \backslash X_{M}^{\Im_{\mathrm{u}}^{w}}$. In this case there exists $h \in\{1, \ldots, p\}$ such that $x \in\left[x_{h}\right], y \notin\left[x_{h}\right]$, and therefore we have that $u_{h}(y)=u_{h}^{\prime}(y)<u_{h}^{\prime}(x)=$ sup $u_{h}(X)+\delta_{h}$. On the other hand, from the fact that $x \precsim_{\mathbf{u}}^{w} y$ is contradictory, we have that there exists $k \in$ $\{1, \ldots, p\}$ such that $u_{k}(y)<u_{k}(x)$. Hence, property (*) is verified also in this case.

(3) $x \in X_{M}^{\Im_{\mathrm{u}}^{w}}$ and $y \in X_{M}^{\Im_{\mathrm{u}}^{w}}$. Clearly, we must have that either $\left(x \sim_{\mathbf{u}}^{w} y\right)$ or $\left(\operatorname{not}\left(x \precsim_{\mathbf{u}}^{w} y\right)\right.$ and $\left.\operatorname{not}\left(y \S_{\mathbf{u}}^{w} x\right)\right)$. In the first case, it is clear that property $(*)$ holds with all equalities on both sides of the equivalence. In the second case, since $x \bowtie_{\mathbf{u}}^{w} y$, there exist $h, k \in$ $\{1, \ldots, m\}$ such that $u_{h}(x)<u_{h}(y)$ and $u_{k}(y)<u_{k}(x)$. On the other hand, the definition of the function $\mathbf{u}^{\prime}$ implies the existence of $h \in\{1, \ldots, p\}$ such that $x \in \arg \max u_{h}$, and, therefore, for that $h$, we have that $u_{h}^{\prime}(y)<u_{h}^{\prime}(x)$. Analogously, there exists $k \in$ $\{1, \ldots, p\}$ such that $y \in \arg \max u_{k}$, and, therefore, for that $k$, we have that $u_{k}^{\prime}(x)<u_{k}^{\prime}(y)$. This consideration completes the proof.

\section{Conclusions}

We approach the multiobjective optimization problem by using the preorders which are naturally associated with the concepts of Pareto optimal and, respectively, weakly Pareto optimal solutions, in the sense that the Pareto optimal and the weakly Pareto optimal solutions are precisely the maximal elements of these preorders. This interpretation gives us the possibility of using all the theorems concerning the maximal elements of the preorders (in particular on compact spaces) in order to guarantee the existence of solutions to the multiobjective optimization problem. This reinterpretation allows us to state a scalarization result under fairly general conditions. Our analysis does not require any particular requirement concerning the functions appearing in the multiobjective optimization problem or the choice set.

\section{Conflicts of Interest}

The authors declare that there are no conflicts of interest regarding the publication of this paper.

\section{References}

[1] K. Miettinen, Nonlinear Multiobjective Optimization, Kluwer Academic Publishers, Norwell, Mass, USA, 1999. 
[2] M. Ehrgott, Multicriteria optimization, vol. 491 of Lecture Notes in Economics and Mathematical Systems, Springer, Berlin, Germany, 2000.

[3] I. Das, "A preference ordering among various pareto optimal alternatives," Journal of Structural Optimization, vol. 18, no. 1, pp. 30-35, 1999.

[4] J. Pietrzak, "A systematic search for Pareto optimum solutions," Journal of Structural Optimization, vol. 17, no. 1, pp. 79-81, 1999.

[5] P. Xidonas, G. Mavrotas, C. Hassapis, and C. Zopounidis, "Robust multiobjective portfolio optimization: a minimax regret approach," European Journal of Operational Research, vol. 262, no. 1, pp. 299-305, 2017.

[6] A. Chateauneuf, M. Mostoufi, and D. Vyncke, "Multivariate risk sharing and the derivation of individually rational Pareto optima," Mathematical Social Sciences, vol. 74, pp. 73-78, 2015.

[7] P. M. Barrieu and G. Scandolo, "General Pareto optimal allocations and applications to multi-period risks," ASTIN Bulletin, vol. 38, no. 1, pp. 105-136, 2008.

[8] A. V. Asimit, V. Bignozzi, K. C. Cheung, J. Hu, and E.-S. Kim, "Robust and Pareto optimality of insurance contracts," European Journal of Operational Research, vol. 262, no. 2, pp. 720-732, 2017.

[9] M. Ehrgott and S. Nickel, "On the number of criteria needed to decide Pareto optimality," Mathematical Methods of Operations Research, vol. 55, no. 3, pp. 329-345, 2002.

[10] R. Bokrantz and A. Fredriksson, "Necessary and sufficient conditions for Pareto efficiency in robust multiobjective optimization," European Journal of Operational Research, vol. 262, no. 2, pp. 682-692, 2017.

[11] C. d'Aspremont and L. Gevers, "Social welfare functionals and interpersonal comparability," Handbook of Social Choice and Welfare, vol. I, Chapter 10, pp. 459-541, 2002.

[12] E. A. Ok, "Utility representation of an incomplete preference relation," Journal of Economic Theory, vol. 104, no. 2, pp. 429449, 2002.

[13] E. Evren and E. A. Ok, "On the multi-utility representation of preference relations," Journal of Mathematical Economics, vol. 47, no. 4-5, pp. 554-563, 2011.

[14] E. Minguzzi, "Normally Preordered Spaces and Utilities," Order, vol. 30, no. 1, pp. 137-150, 2013.

[15] J. C. R. Alcantud, G. Bosi, and M. Zuanon, "Richter-Peleg multi-utility representations of preorders," Theory and Decision, vol. 80, no. 3, pp. 443-450, 2016.

[16] C. Rodríguez-Palmero and J.-L. García-Lapresta, "Maximal elements for irreflexive binary relations on compact sets," Mathematical Social Sciences, vol. 43, no. 1, pp. 55-60, 2002.

[17] G. Bosi and M. . Zuanon, "Maximal elements of quasi upper semicontinuous preorders on compact spaces," Economic Theory Bulletin, vol. 5, no. 1, pp. 109-117, 2017.

[18] V. V. Podinovski, "Non-dominance and potential optimality for partial preference relations," European Journal of Operational Research, vol. 229, no. 2, pp. 482-486, 2013.

[19] V. V. Podinovski, "Potential optimality of pareto optima," in Proceedings of the 1st International Conference on Information Technology and Quantitative Management, ITQM 2013, pp. 1107-1112, China, May 2013.

[20] D. J. White, "Notes in decision theory: optimality and efficiency II," European Journal of Operational Research, vol. 4, no. 6, pp. 426-427, 1980.

[21] M. K. Richter, "Revealed Preference Theory," Econometrica, vol. 34, no. 3, pp. 635-645, 1966.
[22] B. Peleg, "Utility functions for partially ordered topological spaces," Econometrica, vol. 38, pp. 93-96, 1970.

[23] J. Ward Jr., "Partially ordered topological spaces," Proceedings of the American Mathematical Society, vol. 5, pp. 144-161, 1954.

[24] T. C. Bergstrom, "Maximal elements of acyclic relations on compact sets," Journal of Economic Theory, vol. 10, no. 3, pp. 403404, 1975.

[25] J. C. Alcantud, "Characterization of the existence of maximal elements of acyclic relations," Economic Theory, vol. 19, no. 2, pp. 407-416, 2002. 


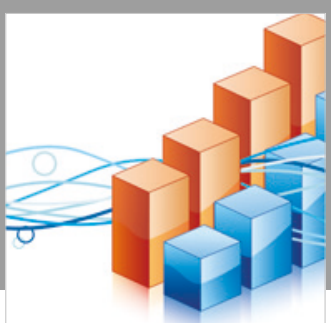

Advances in

Operations Research

\section{-n-m}
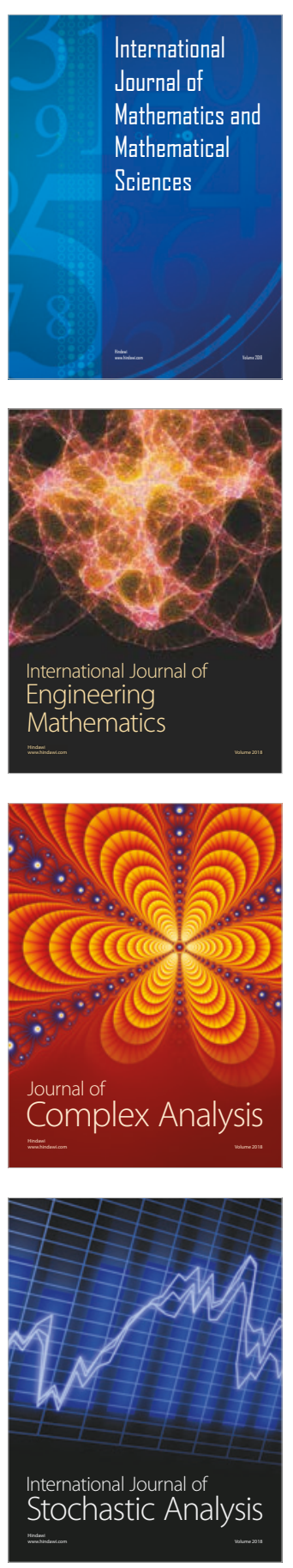
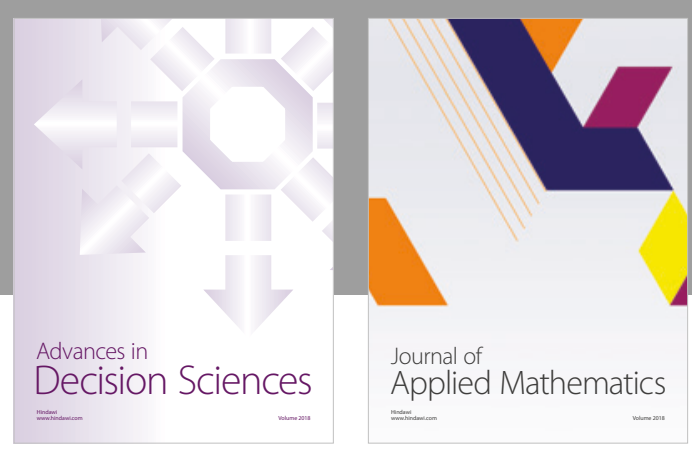

Journal of

Applied Mathematics
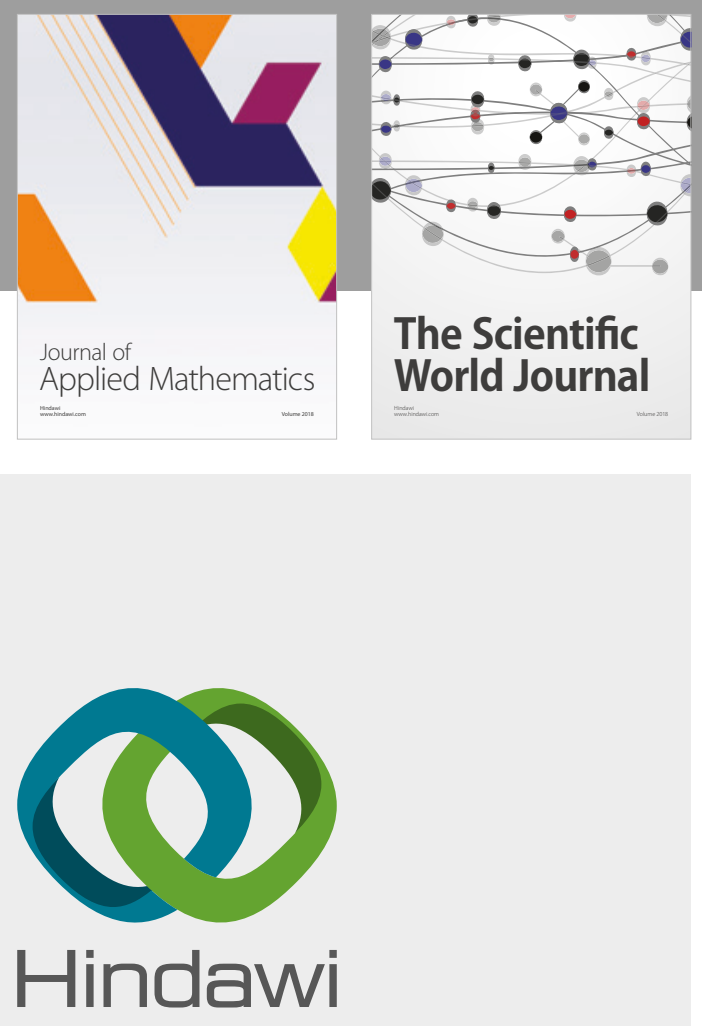

Submit your manuscripts at

www.hindawi.com

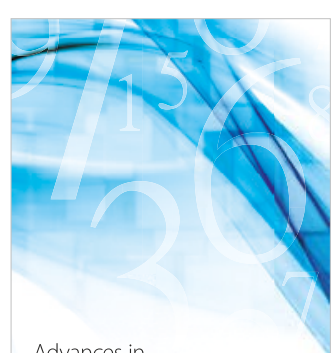

Advances in
Numerical Analysis
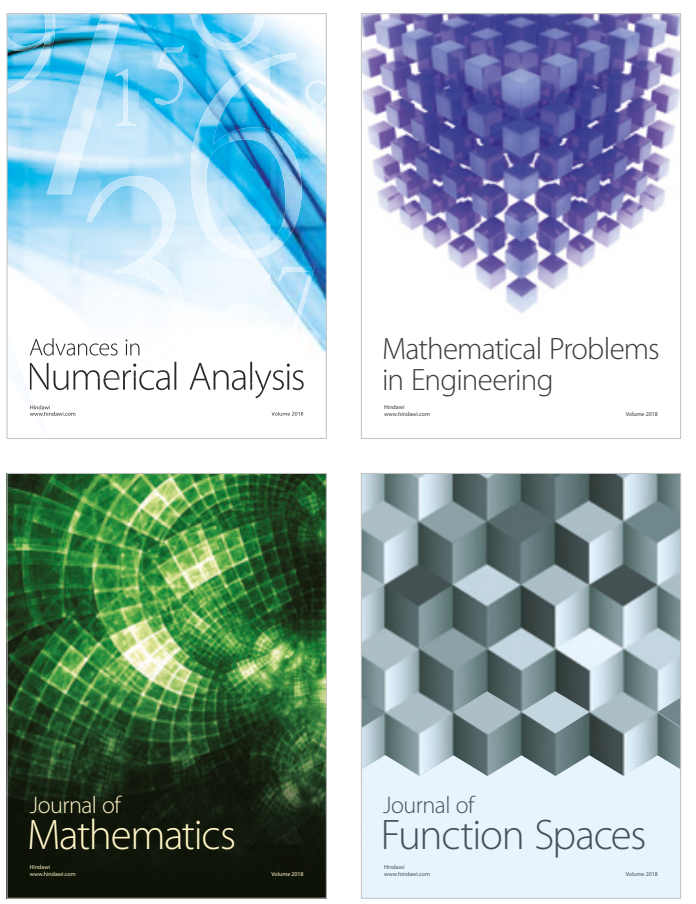

Mathematical Problems in Engineering

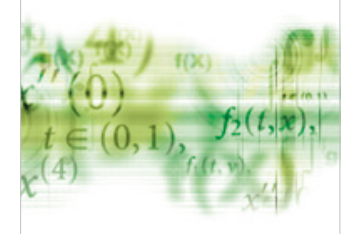

International Journal of

Differential Equations

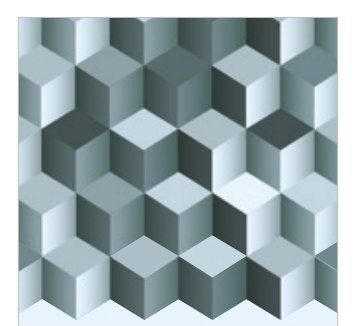

Journal of

Function Spaces

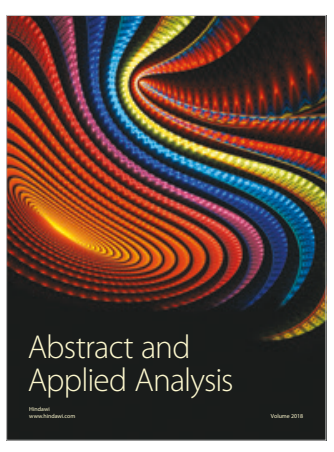

The Scientific

World Journal

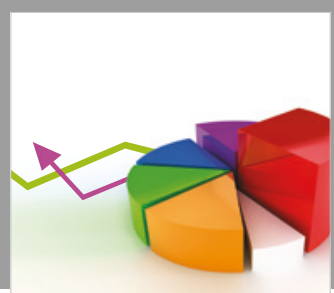

Journal of

Probability and Statistics
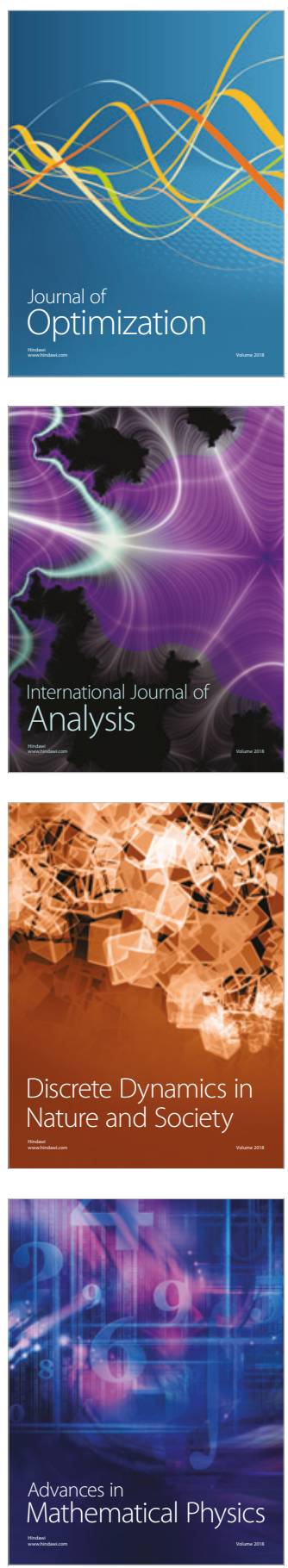